\title{
An Observation of Healthcare Professions Students' Perceptions During the COVID-19 Pandemic
}

\author{
Anette $\mathrm{Wu}^{1}{ }^{1}$ - Vinay Maddula ${ }^{1} \cdot$ Ann Chen $\mathrm{Xi} \mathrm{Yu}^{2} \cdot$ Rahul Goel $^{3} \cdot$ Haruna Shimizu $^{1} \cdot$ Chung-Liang Chien ${ }^{4}$. \\ Richard Wingate $^{3} \cdot$ Mandeep Gill Sagoo $^{3} \cdot$ Heike Kielstein $^{5} \cdot$ Hannes Traxler $^{6} \cdot$ Cecilia Brassett $^{7}$. Jens Waschke ${ }^{8}$. \\ Fransziska Vielmuth ${ }^{8} \cdot$ Kevin Keay $^{2} \cdot$ Mina Zeroual $^{9} \cdot$ Takeshi Sakurai $^{10} \cdot$ Jorgen Olsen $^{11} \cdot$ Salma El-Batti $^{12}$. \\ Suvi Viranta-Kovanen ${ }^{13}$. Shuji Kitahara ${ }^{14}$. Carol Kunzel $^{1} \cdot$ Paulette Bernd $^{1} \cdot$ Geoffroy P.J.C. Noel $^{9}$
}

Accepted: 3 February 2021 / Published online: 18 February 2021

(c) International Association of Medical Science Educators 2021

\begin{abstract}
This study conveys preclinical healthcare professions students' sentiments at 14 universities during the 2020 COVID-19 pandemic. Essays about students' thoughts and experiences were thematically sorted and revealed a variety of sentiments spanning from positive (e.g., pride, respect) to the more negative (e.g., anxiety, guilt, disappointment, anger). Themes revealed respect for the healthcare profession, but also the realization of its limitations, sacrifices, and risks. Healthcare profession educators need to be aware that the COVID-19 pandemic has affected students emotionally and may have long-term effects on the global healthcare profession. This study can serve as a historic documentation of how this generation of students felt and adds to the literature on how the pandemic affected the healthcare profession.
\end{abstract}

Keywords COVID-19 $\cdot$ Healthcare professions education $\cdot$ Student experiences $\cdot$ Student emotions $\cdot$ International comparison

Anette $\mathrm{Wu}$

aw2342@caa.columbia.edu

Columbia University, New York, NY, USA

2 University of Sydney, Sydney, Australia

3 King's College, London, UK

4 National Taiwan University, Taipei, Taiwan

5 Martin Luther University, Halle-Wittenberg, Germany

6 Medical University of Vienna, Vienna, Austria

7 University of Cambridge, Cambridge, UK

8 Ludwig Maximilian University, Munich, Germany

9 McGill University, Montreal, QC, Canada

10 Kyoto University, Kyoto, Japan

11 University of Copenhagen, Copenhagen, Denmark

12 University of Paris Descartes, Paris, France

13 University of Helsinki, Helsinki, Finland

14 Tokyo Women's Medical University, Tokyo, Japan

\section{Background}

The global COVID-19 pandemic has significantly impacted healthcare professions education on many levels [1]university campuses closed [2], classes were moved to online formats and/or were significantly altered [3, 4], clinical training was interrupted and impacted [5-7], and in some countries, senior medical students graduated early to work on the frontlines [8].

To date, there is no clear understanding about how this pandemic impacts various aspects of learner education; including their emotions, learning activities, and career aspirations-in particular, from a global perspective.

As the pandemic affects countries and regions differently, the experiences and impact on the trainees worldwide can vary, and thus have different effects on healthcare globally.

The goal of this study was to capture students' perceptions, experiences, and emotions at 14 universities in 11 countries, during the 2020 COVID-19 pandemic.

Understanding how worldwide health events can impact students in different parts of the world is important for healthcare professions educators and will form the baseline of a historical context for how 
global issues can impact learner experiences, as it can have long-term consequences for young students at both the personal and professional levels, and therefore, on healthcare professions education and the healthcare profession at large.

The compilation of these perceptions serves as a historic documentation of students' thoughts during an unprecedented global pandemic, adds to the current literature on the effect of the COVID-19 pandemic on the healthcare profession, and serves as a baseline for longitudinal research on the professional careers of this particular generation of students.

\section{Activity}

The participants in this study were preclinical healthcare professions students (i.e., medical and dental students) who volunteered in an online international student exchange program [9].

The students were asked to share their experiences during the COVID-19 pandemic in small international focus groups. Subsequently, they submitted individual essays about their thoughts, guided by open-ended questions (Table 1).

The written assignments were submitted anonymously, collected via Google Forms $\odot$, and analyzed for thematic content using a general inductive approach [10]. Five project team researchers of different ages, backgrounds, and ethnicities (A.W., V.M., R.G., C.X.Y., and H.S.) independently reviewed each submission. Specifically, the coders received anonymous essays that were blinded for originating school, gender, and age-only after the thematic coding was completed was this information revealed. The assignments were coded in two phases. In phase 1, the coders read all the essays and identified themes for each of the guided questions. All coders agreed on the themes before proceeding to the next step. In the second phase, the responses were then categorized into the coded themes using Excel@ The project team met regularly to resolve discrepancies and achieve consensus. Discrepancies included disagreement on interpretation or categorization of the content. Consensus was achieved via majority vote. If no consensus was achieved, the essay was excluded. Once all the essays were coded, the written pieces were identified and sorted by schools.

Due to disproportionate representation from each school, a comparison between schools was not possible, but striking differences were noted (i.e., when a theme was identified for all schools).

All assignments were collected between June 15 and July 15, 2020. Written consent was obtained as part of the program participation process.

Ethical approval was obtained from Columbia University (IRB No. AAA0003715), McGill University (IRB Study No.
A07-E54-17B), and the National Taiwan University (IRB No. 202001069 W).

\section{Results}

\section{Student Demographics}

A total of 81 students from 14 universities participated (Table 2). Eighty written assignments were received; essays were obtained from 69 medical and 11 dental students.

The results of the study focused on four of the selected guided questions listed in Table 1. Table 1 depicts representative sample quotes for each theme, subtheme, and sub-subtheme.

Overall, the students' responses revealed several overarching themes, and the majority of students each addressed a variety of emotions. The themes were grouped into positive and negative emotions. Furthermore, some themes were subdivided into subthemes and sub-subthemes.

\section{Sentiments}

A positive emotion such as pride appears to be a sentiment that was present in many schools, with different subsubthemes (e.g., proud to be a member of a school, a member of a healthcare profession, a member of the healthcare field, a citizen of a country). Negative emotions, such as fear and anxiety caused by the pandemic, were present in all schools regardless of country (e.g., fear of the disease, of the pandemic in general, for loved ones, for the healthcare profession, and anxiety about the future and the impact on education). Anger and frustration were commonly found in select countries, subdivided into three sub-subthemes (e.g., anger at the government, at others, at the situation). Students also expressed an underlying level of guilt.

Overall, it appears that students in the USA and the UK did experience more negative emotions (e.g., fear, anger, frustration, and disappointment), when compared for example with Denmark, Finland, Germany, and Taiwan. Positive emotions in the USA and the UK focused on pride in being a healthcare professional.

\section{Changes in Outlook on the Healthcare Profession}

Respect for, and importance of, the healthcare profession dominated. Students also recognized the importance of good leadership in healthcare. Realization of the limitations of the profession, including sacrifice, risks, and undervaluation of the profession, was also found. 


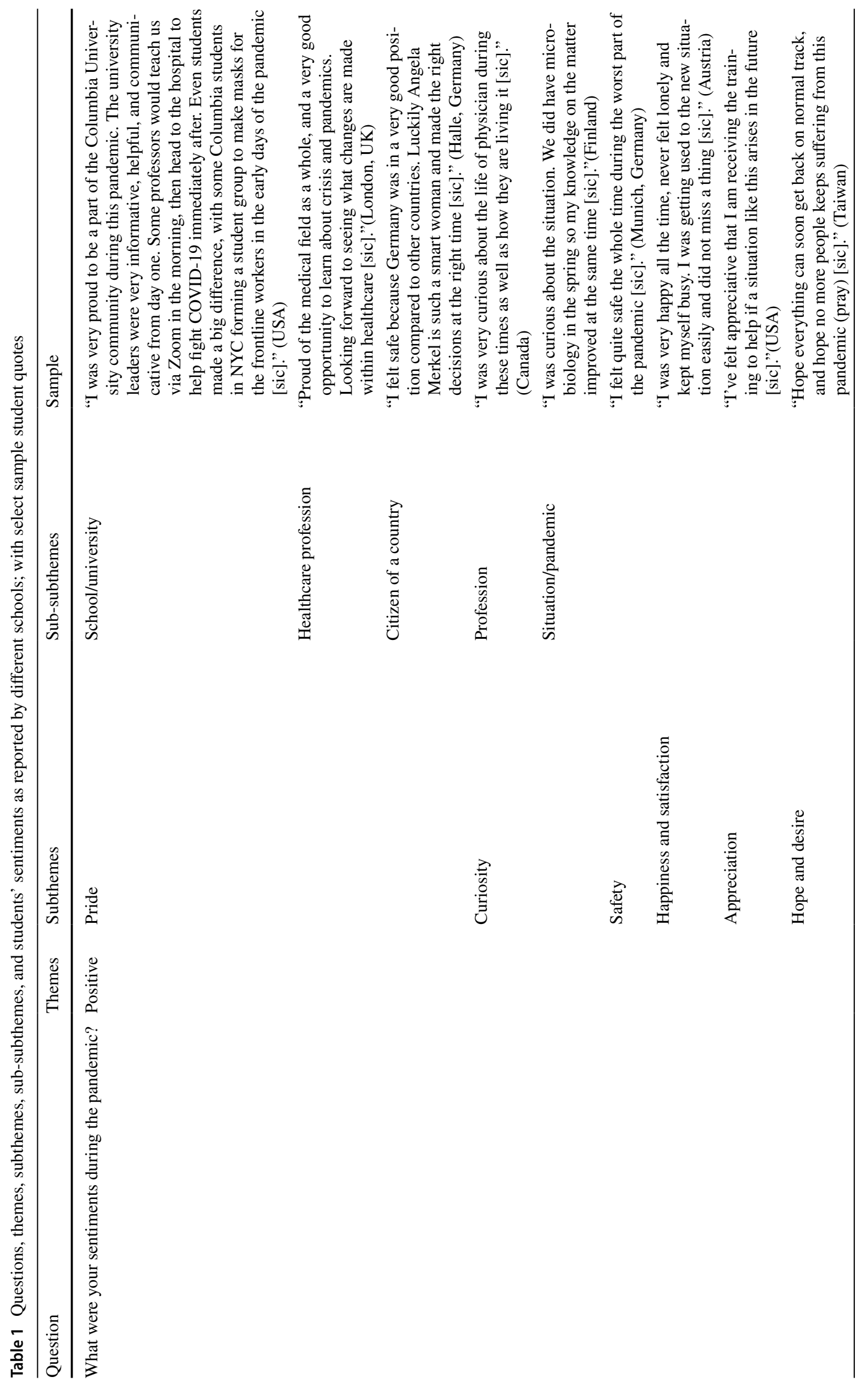




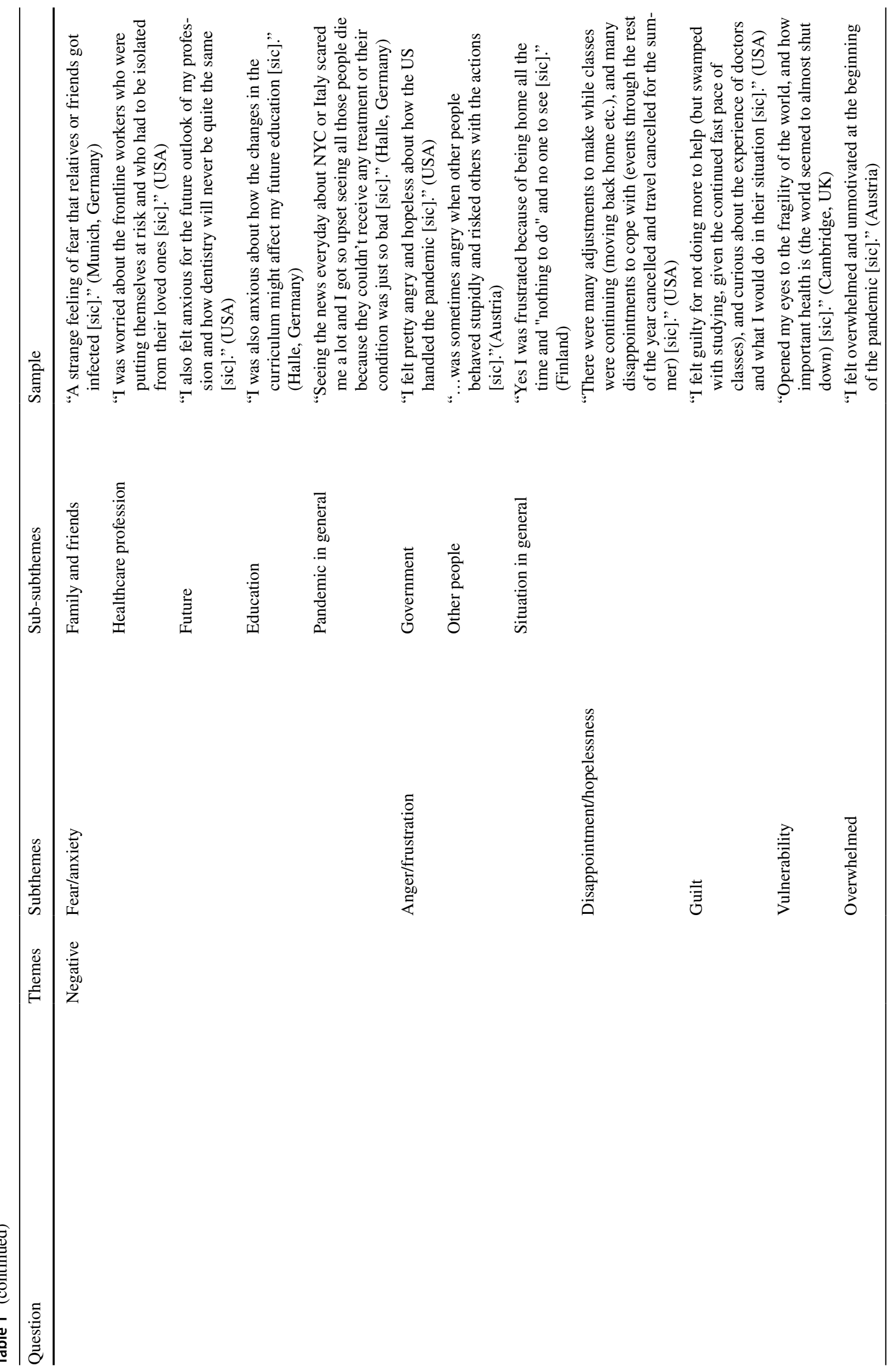




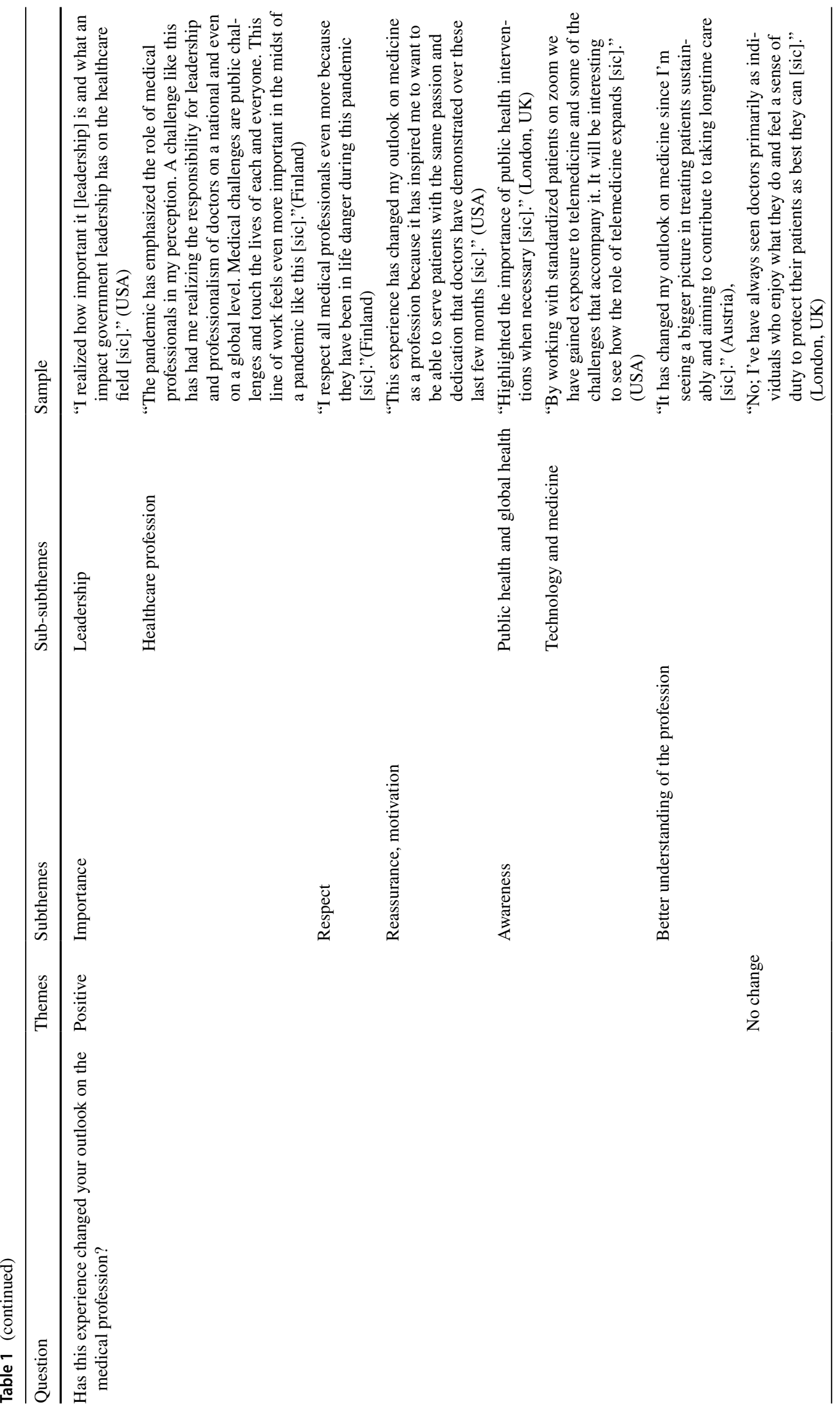




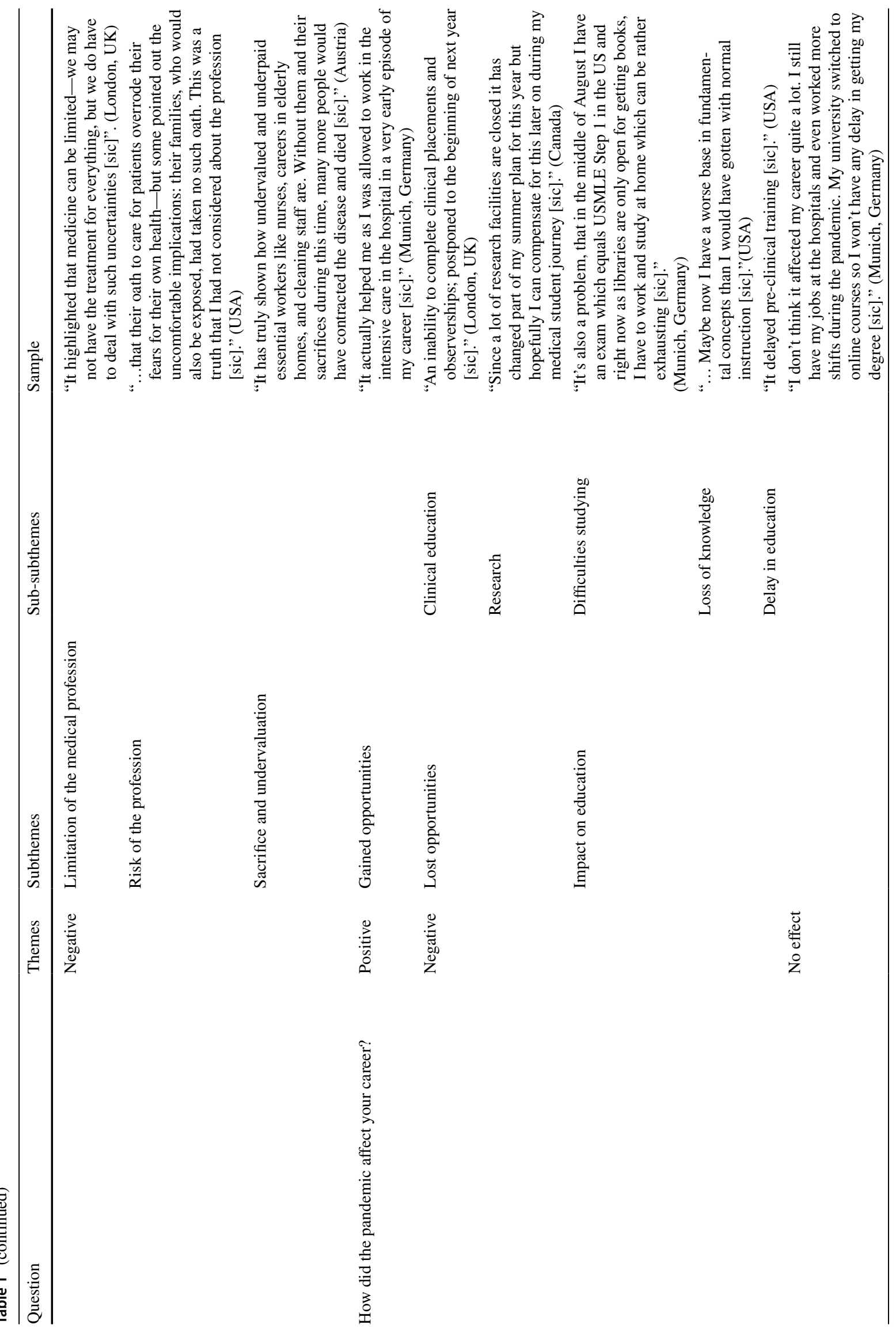




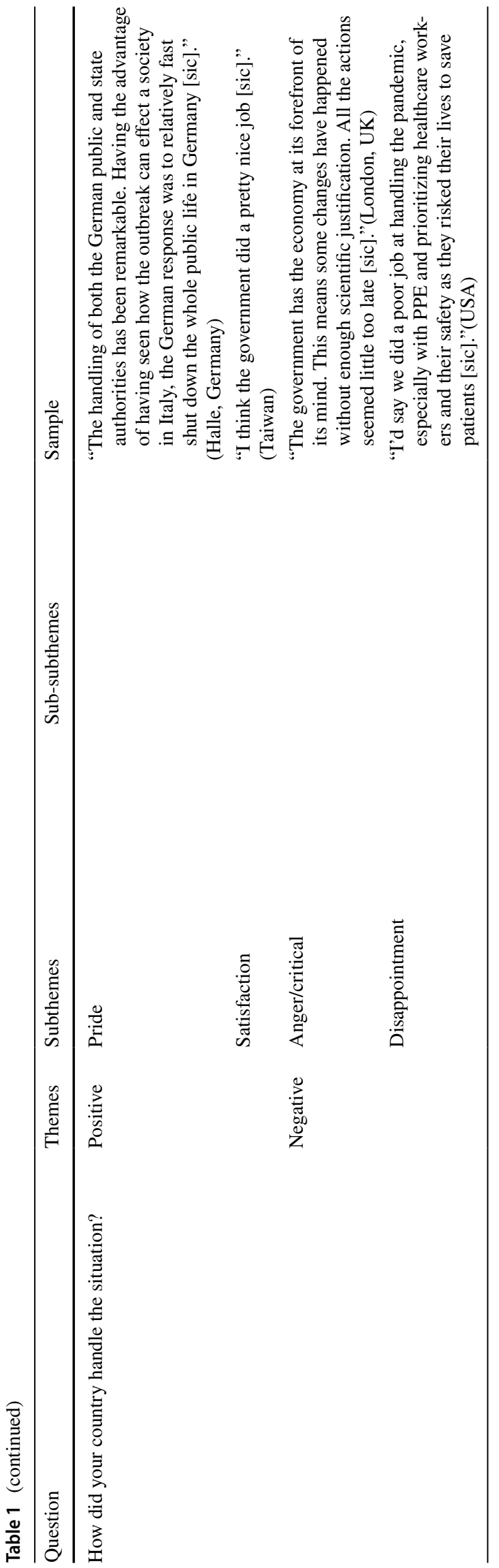

In some students, the pandemic experience had no effect on their view of the profession.

\section{Impact on Career}

The students felt that the pandemic affected their careers, in positive and negative ways.

Positive effects included emergence of new opportunities. Negative effects included lost opportunities, with subsubthemes of loss of clinical education and research opportunities. Students perceived that their education was impacted, including difficulty studying, loss of knowledge, and delay in education.

For a number of students, the pandemic had little or no impact.

\section{Country's Handling of the Situation}

Positive and negative feelings were associated with how the pandemic was managed in the respective countries. Students reported having positive feelings such as pride and satisfaction. Negative feelings found in select countries (i.e., USA, UK) included anger/criticism and disappointment. A few students had mixed positive and negative emotions regarding their country. When reviewing emerging themes, it became evident that countries that did not function well during the pandemic (summer 2020) evoked negative feelings, whereas in countries that did perform well, more students had something positive to report.

\section{Discussion}

In the present study, the authors reviewed the perceived impact of the recent COVID-19 outbreak on healthcare profession students from different parts of the world - in an observational snapshot, at a given point in time (June 15-July 15, 2020), to document the experiences of this generation of students.

Changes in study environments and school curricula, social isolation due to lockdown or quarantine, restriction of travel opportunities, and reshaping of human interaction have profoundly impacted individual and societal behaviors.

Unsurprisingly, a wide array of emotions was found that requires the attention of healthcare profession educators.

Independent of how severely the COVID-19 pandemic affected the students' home countries at the time, all universities had representations of students' fear and anxiety. Major disasters and pandemics in general can have a lasting psychological effect on a young person [11-13], and medical students' reactions to natural disasters have been reported [14-19]. This report confirms the findings about emotions during pandemics in general [20]; particularly, during the COVID-19 pandemic [20-23]. These emotions may lead to 
Table 2 Participating universities, with student demographics. Fourteen schools participated in the program, and assignments were received from 13 schools. One school did not provide any written

\begin{tabular}{|c|c|c|c|c|c|c|c|}
\hline \multirow[t]{2}{*}{ University } & \multicolumn{3}{|c|}{ Gender } & \multicolumn{3}{|l|}{ Ages } & \multirow[t]{2}{*}{ Total } \\
\hline & Male & Female & Undisclosed & Under 20 & $\begin{array}{l}\text { Between } 20 \\
\text { and } 25\end{array}$ & Above 25 & \\
\hline Colombia University, New York, USA & 6 & 15 & 0 & 1 & 15 & 5 & 21 \\
\hline Kings College London, London, UK & 3 & 1 & 0 & 3 & 1 & 0 & 4 \\
\hline Kyoto University, Kyoto, Japan & 0 & 1 & 0 & 0 & 1 & 0 & 1 \\
\hline Ludwig Maximilians University, Munich, Germany & 0 & 5 & 0 & 2 & 3 & 0 & 5 \\
\hline Martin Luther University, Halle-Wittenberg, Germany & 2 & 4 & 0 & 0 & 6 & 0 & 6 \\
\hline McGill University, Montreal, Canada & 0 & 3 & 1 & 0 & 4 & 0 & 4 \\
\hline Medical University of Vienna, Vienna, Austria & 2 & 7 & 0 & 3 & 4 & 2 & 9 \\
\hline National Taiwan University, Taipei, Taiwan & 7 & 4 & 0 & 1 & 7 & 3 & 11 \\
\hline Tokyo Women's Medical University, Tokyo, Japan & 0 & 1 & 0 & 0 & 0 & 1 & 1 \\
\hline University of Cambridge, Cambridge, UK & 1 & 4 & 0 & 1 & 3 & 1 & 5 \\
\hline University of Copenhagen, Copenhagen, Denmark & 1 & 1 & 0 & 0 & 2 & 0 & 2 \\
\hline University of Helsinki, Helsinki, Finland & 0 & 8 & 0 & 0 & 7 & 1 & 8 \\
\hline University of Paris, Paris, France & 1 & 2 & 0 & 2 & 1 & 0 & 3 \\
\hline University of Sydney, Sydney, Australia & 0 & 1 & 0 & 0 & 1 & 0 & 1 \\
\hline Total & & & & & & & 81 \\
\hline
\end{tabular}

submissions but participated in the small group discussions about the COVID-19 pandemic mental health issues, such as post-traumatic stress disorder (PTSD), depression, and anxiety, but can also result in positive outcomes such as personal growth and resilience in the long term [14, 17]. Furthermore, during disasters, media exposure alone can have a psychological effect on students' emotions [24]. This could explain the ubiquitous presence of fear and anxiety among students who live in countries that were minimally affected by the pandemic at the time. Therefore, it may be advisable for schools to offer crisis support to students of this generation, as previously suggested [25].

Professional identity formation has recently been investigated and is a several-phase process [26]. It was reassuring to see that the severity of the pandemic was not associated with students having doubts about their choice of profession.

The finding that students felt that the pandemic made them proud to be in the healthcare profession is of importance for the future of healthcare.

It appears that a significant number of students reflected on challenges inherent to the healthcare profession (e.g., danger, risk, sacrifice, importance of leadership, global health). This finding is of importance as it may prompt changes in their future career paths.

Although too early to evaluate the impact on their healthcare professions education, the students were concerned with negative effects on their careers. However, many of them were hopeful that over the long term, a delay in education/training would not be of importance. The authors suggest that universities should consider offering these students additional research and training opportunities to make up for the time lost during the pandemic, so that they can remain competitive with previous cohorts.

It will be important to follow-up with this generation of students and to assess the educational outcomes of this particular cohort. This information is particularly important, as the role of online learning might be increasing in higher education.

Notably, despite the different effects of the pandemic on their home countries, students in all countries reported a mix of positive and negative emotions, indicating the complexity of their reflections. For that reason, as part of the study, the authors included the students' opinions of how their countries fared-to underline the intricacy of what influenced their feelings.

Although the voluntary nature of the program, the focus on preclinical students, the lack of a control group, and the small number of participants limit the generalizability of the results, the authors conclude that overall, the pandemic affected healthcare professions students. Although a likely possibility, it is too early to surmise if this experience will have a long-term effect on these students' careers. This observational study can serve as a historic documentation of how students of this particular generation felt during a global crisis, can serve as a resource for further comparative investigations, and supports healthcare professions educators in addressing future problems. 


\section{Future Directions}

After this initial observational study, focused qualitative and quantitative research with more students is underway, to investigate the impact of the pandemic on the students' mental health and professional careers.

Acknowledgements The authors would like to thank the participating student leaders for their help in the international program-Adedeji Adeniyi (Columbia University, USA), Anita Chen (National Taiwan University, Taiwan), Erin Fitzsimmons-West (University of Cambridge, UK), Jasmine Singh (King's College London, UK), and Kerstin Saraci (Ludwig Maximilians University, Munich, Germany), and Dr. Michael Fortgang for helpful review of the manuscript.

\section{Declarations}

Conflict of Interest The authors declare that they have no conflict of interest.

\section{References}

1. Rabe ASM, Cheung W, Lucero-Prisno D. COVID-19 and health professions education: a $360^{\circ}$ view of the impact of a global health emergency. Med Ed Publish. 2020;9(1):148.

2. Sahu P. Closure of universities due to coronavirus disease 2019 (COVID-19): impact on education and mental health of students and academic staff. Cureus. 2020;12(4):e7541.

3. Singh K, Srivastav S, Bhardwaj A, Dixit A, Misra S. Medical education during the COVID-19 pandemic: a single institution experience. Indian Pediatr 2020.

4. Moszkowicz D, Duboc H, Dubertret C, Roux D, Bretagnol F. Daily medical education for confined students during COVID-19 pandemic: a simple videoconference solution. Clin Anat. 2020.

5. Theoret C, Ming X. Our education, our concerns: the impact on medical student education of COVID-19. Med Educ. 2020.

6. Stokes DC. Senior medical students in the COVID-19 response: an opportunity to be proactive. Acad Emerg Med. 2020;27(4):343-5.

7. Calhoun KE, Yale LA, Whipple ME, Allen SM, Wood DE, Tatum RP. The impact of COVID-19 on medical student surgical education: implementing extreme pandemic response measures in a widely distributed surgical clerkship experience. Am J Sur. 2020.

8. Iacobucci G. Covid-19: medical schools are urged to fast-track final year students. BMJ. 2020;368:m1064.

9. Wu A, Maddula V, KIeff MR, Kunzel C. An online program to improve international collaboration, intercultural skills, and research knowledge. J Dent Educ. 2020 1-4.

10. Thomas DR. A general inductive approach for analyzing qualitative evaluation data. Am J Eval. 2006;27(2):237-46.
11. Oh JK, Lee MS, Bae SM, Kim E, Hwang JW, Chang HY, et al. Psychiatric symptoms and clinical diagnosis in high school students exposed to the Sewol Ferry disaster. J Korean Med Sci. 2019;34(5):e38.

12. Jensen GD. Emotional response of students to a political crisis. Am J Psychiatry. 1971;128(3):356-8.

13. Clark J. Fear of SARS thwarts medical education in Toronto. BMJ. 2003;326(7393):784.

14. Kaye-Kauderer HP, Levine J, Takeguchi Y, Machida M, Sekine $\mathrm{H}$, Taku K, et al. Post-traumatic Growth and resilience among medical students after the March 2011 disaster in Fukushima Japan. Psychiatry Q. 2019;90(3):507-18.

15. Mincin J, Hansen R. Disaster mental health in higher education: a review. J Emerg Manag (Weston, Mass). 2019;17(3):217-24.

16. Taku K, Prioleau PG, Anderson DS, Takeguchi Y, Sekine H, Maeda M, et al. Medical student reactions to disaster after the 2011 Great East Japan Earthquake: motivation and posttraumatic growth. Psychiatry Q. 2018;89(4):1007-18.

17. Carter F, Bell C, Ali A, McKenzie J, Boden JM, Wilkinson T, et al. Predictors of psychological resilience amongst medical students following major earthquakes. N Z Med J. 2016;129(1434):17-22.

18. Liu S, Lu L, Bai ZZ, Su M, Qi ZQ, Zhang SY, et al. Post-traumatic stress and school adaptation in adolescent survivors five years after the 2010 Yushu Earthquake in China. Int J Environ Res Public Health. 2019;16(21).

19. Liu D, Fu L, Jing Z, Chen C. Post-traumatic stress disorder and it's predictors among Tibetan adolescents 3years after the high-altitude earthquake in China. Arch Psychiatr Nurs. 2016;30(5):593-9.

20. Huremovic D. Psychiatry of pandemics. A mental health response to infection outbreak. Huremović D, editor. Switzerland: Springer, Cham; 2019

21. Cao W, Fang Z, Hou G, Han M, Xu X, Dong J, et al. The psychological impact of the COVID-19 epidemic on college students in China. Psychiatry Res. 2020;287:112934.

22. Shaw SCK. Hopelessness, helplessness and resilience: the importance of safeguarding our trainees' mental wellbeing during the COVID-19 pandemic. Nurse Educ Pract. 2020;44:102780.

23. Wu W, Zhang Y, Wang P, Zhang L, Wang G, Lei G, et al. Psychological stress of medical staffs during outbreak of COVID19 and adjustment strategy. J Med Virol. 2020.

24. Yeung NCY, Lau JTF, Yu NX, Zhang J, Xu Z, Choi KC, et al. Media exposure related to the 2008 Sichuan Earthquake predicted probable PTSD among Chinese adolescents in Kunming, China: a longitudinal study. Psychol Trauma Theory Res Pract Policy. 2018;10(2):253-62.

25. Wald HS. Optimizing resilience and wellbeing for healthcare professions trainees and healthcare professionals during public health crises - practical tips for an 'integrative resilience' approach. Med Teach. 2020;1-12.

26. Ward J, Randall V. From college graduate to physician: professional identity formation. Med Ed Publish. 2020;9(1):180.

Publisher's Note Springer Nature remains neutral with regard to jurisdictional claims in published maps and institutional affiliations. 